\title{
Effects of Passive Lower Limb and Trunk Exercises and Diaphragm Breathing Exercise on Intestinal Movement
}

\author{
Tomoyuki Morisawa, PT, MSc ${ }^{1)}$, Tetsuya Takahashi, PT, $\mathrm{PhD}^{2)}$, Shinichi Nishi, MD, $\mathrm{PhD}^{3)}$ \\ 1) Department of Physical Therapy, School of Rehabilitation, Hyogo University of Health Sciences: \\ 1-3-6 Minatoshima, Chuo-ku, Kobe-shi, Hyogo 650-8530, Japan. TEL: +81 78-304-3181, FAX: +81 \\ 78-304-8211,E-mail: morisawa@huhs.ac.jp \\ 2) Department of Physical Therapy, School of Health Sciences, Tokyo University of Technology \\ 3) Intensive Care Unit, Hyogo College of Medicine
}

\begin{abstract}
Purpose] This study examined the effects of passive lower limb and trunk exercises (PELT) and diaphragmatic breathing exercise (DBE) on intestinal motility in order to devise new techniques to effectively encourage intestinal motility. [Subjects and Methods] The subjects were 18 healthy adult males. Their peristaltic sounds were recorded at rest. Subjects then randomly received either PELT or performed DBE for 10 min, and their peristaltic sounds were recorded afterwards. Several days later, the sounds were recorded again. An electronic stethoscope with recording capability was used to record peristaltic sounds. Recorded peristaltic sounds were passed through an amplifier and a data acquisition and analysis system and uploaded to a PC. The integral of the primary frequency range of sounds; was calculated every $10 \mathrm{sec}$. [Results] Following PELT, peristaltic sounds increased significantly from the levels at rest, but significant differences between peristaltic sounds after DBE and sounds at rest were not noted. For $20 \mathrm{sec}$ after either intervention, sounds increased significantly compared to the sounds at rest, but subsequently significant differences were not noted. [Conclusion] Both techniques had an immediate effect but no sustained effect. In future clinical settings, interventions should be performed several times for patients with decreased intestinal motility.

Key words: Bowel sounds, Passive exercise, Diaphragmatic breathing
\end{abstract}

(This article was submitted Aug. 23, 2012, and was accepted Oct. 5, 2012)

\section{INTRODUCTION}

One reason patients are unable to leave their beds soon after developing an illness or undergoing surgery is a decrease in intestinal motility. Decreased intestinal motility can lead to symptoms such as abdominal discomfort, abdominal pain, nausea, and decreased intestinal motility while a patient is in the ICU can negatively impact the patient overall, affecting processes such as respiration, circulation, and metabolism. Decreased intestinal motility can also affect the prognosis for patients with severe ileus.

Previously reported techniques for encouraging intestinal motility include hot compresses ${ }^{1)}$, compression ${ }^{2}$, abdominal massage $^{3,4)}$, low-frequency stimulus ${ }^{5)}$, and acupressure ${ }^{6}$, and these techniques are widely used in clinical settings. These techniques are considered to enhance circulation in the abdomen and to affect parasympathetic nervous activity, but the outcomes of these techniques is unclear. The reality is that there is a dearth of scientific proof regarding the effectiveness of these techniques. New techniques should be devised to effectively encourage intestinal motility in patients who are in bed and unable to exercise independently or leave their beds due to therapeutic constraints.

Stretching of the intestines and increased intra-abdominal pressure are known to be crucial for encouraging intestinal motility. In the current study, passive lower limb and trunk exercises (PELT) were used to stretch the intestines while diaphragmatic breathing exercise (DBE) was used to increase intra-abdominal pressure. This study examined the effects of PELT and DBE on intestinal motility in order to devise new techniques for effectively encouraging intestinal motility in patients in bed.

\section{SUBJECTS AND METHODS}

The subjects were 18 healthy adult males with no previous history of gastrointestinal, respiratory, bone or joint disorders. Subjects had a mean age of $20.8 \pm 0.8$ years (20-22 years of age) and a mean BMI of $21.1 \pm 2.3$ (17.926.1). This study was fully explained to subjects, who then consented to participation in writing. In addition, this study was reviewed and approved by the Ethics Committee of the Hyogo University of Health Sciences (No. 09026).

Subjects were instructed to avoid excessive exercise or overeating on the day prior to testing and to go about their normal activities. On the day of the test, subjects consumed a similar meal for breakfast and lunch and then fasted. Subjects assembled in the laboratory $1 \mathrm{hr}$ after lunch. To 
eliminate the effects of changes in posture, subjects rested on a bed in the supine position for $15 \mathrm{~min}$, and then their peristaltic sounds at rest were recorded. Subjects then randomly received either PELT or performed DBE for $10 \mathrm{~min}$, and their peristaltic sounds were recorded for $5 \mathrm{~min}$ afterwards. Several days later, the sounds were recorded again. PELT was administered with subjects in the supine position. One leg was passively flexed and the full flexion position was maintained for five seconds. Each subject was administered this passive exercise ten times for each leg. In addition to leg flexion, right and left trunk rotations were also alternately performed . DBE was performed in Fowler's position. The subjects were asked to perform diaphragm breathing emphatically for ten minutes.

Bowel sounds are generated by contractions of the alimentary tract, and the mixing of gaseous and liquid contents ${ }^{7}$. Recording bowel sounds with a stethoscope and then analyzing them is simple, easy, and minimally invasive, so this procedure has often been used to assess intestinal motility ${ }^{8-11)}$. This procedure was used in the current study.

An electronic stethoscope with recording capability (Fuji Medical Co, Memory Stethoscope F-812) was used to record bowel sounds at the midpoint of the line connecting the umbilical region and the right anterior superior iliac spine. Anatomically, the recording site roughly corresponds to the area of the ileocecal valve, and this is the area where postprandial peristaltic sounds are normally listened to. Recorded peristaltic sounds were passed through an amplifier and fed to a data acquisition and analysis system (ADInstruments, PowerLab 2/26). Waveforms of the peristaltic sounds were converted from analog to digital signals using PowerLab and uploaded to a PC. Frequency analysis was performed at $100-400 \mathrm{~Hz}$, the primary frequency range of peristaltic sounds ${ }^{7}$, and the integral was calculated every 10 sec. The t-test was used to compare sounds, while Dunnett's test was used to determine the changes over time. The level of significance used for both methods was $\mathrm{p}<0.05$.

\section{RESULTS}

Following PELT, peristaltic sounds increased in 14 of 18 patients, and those sounds increased significantly (1.31 \pm $0.49 \mathrm{mV} \cdot \mathrm{sec}$ vs. $1.49 \pm 0.48 \mathrm{mV} \cdot \mathrm{sec}, \mathrm{p}=0.004)$ compared to the same sounds at rest. Following DBE, 8 of 18 patients had increased peristaltic sounds compared to the sounds at rest. Significant differences between these sounds after DBE and sounds at rest were not noted $(1.44 \pm 0.63 \mathrm{mV} \cdot \mathrm{sec}$ vs. 1.36 $\pm 0.45 \mathrm{mV} \cdot \mathrm{sec}, \mathrm{ns})$. For $20 \mathrm{sec}$ after PELT, the sounds were significantly greater than the sounds at rest $(p<0.05)$. After $20 \mathrm{sec}$, there were no significant differences in the sounds, but the sounds after PELT were greater than the sounds at rest. For $20 \mathrm{sec}$ after DBE, the sounds were significantly greater than the sounds at rest $(\mathrm{p}<0.01)$, but afterwards the sounds were essentially the same as they were at rest (Table 1).

\section{DISCUSSION}

Previously reported techniques for encouraging intes-
Table 1. Temporal changes of PELT and DBE

\begin{tabular}{lll}
\hline & PELT $(\mathrm{mV} \cdot \mathrm{sec})$ & $\mathrm{DBE}(\mathrm{mV} \cdot \mathrm{sec})$ \\
\hline rest & $0.044 \pm 0.003$ & $0.047 \pm 0.006$ \\
after 20 sec & $0.069 \pm 0.035^{*}$ & $0.068 \pm 0.027^{* *}$ \\
after 40 sec & $0.059 \pm 0.031$ & $0.050 \pm 0.022$ \\
after 60 sec & $0.048 \pm 0.015$ & $0.047 \pm 0.037$ \\
after 120 sec & $0.047 \pm 0.016$ & $0.044 \pm 0.028$ \\
after 180 sec & $0.048 \pm 0.019$ & $0.043 \pm 0.018$ \\
after 240 sec & $0.047 \pm 0.021$ & $0.044 \pm 0.028$ \\
after 300 sec & $0.045 \pm 0.019$ & $0.042 \pm 0.011$ \\
\hline
\end{tabular}

average $\pm \mathrm{SD}$, Dunnett's test. ${ }^{*} \mathrm{p}<0.05,{ }^{* *} \mathrm{p}<0.01$

tinal motility include heat therapy, compression, massage, and low-frequency stimulus. These therapeutic techniques are widely used in clinical settings, but the reality is that evidence of the effectiveness of these techniques is lacking. There is a pressing need to devise new techniques to effectively encourage intestinal motility in patients who are in bed and unable to exercise independently or leave their beds due to therapeutic constraints.

Following PELT, peristaltic sounds were found to increase significantly. The intestines consist primarily of smooth muscle. When visceral smooth muscle is stretched, its membrane potential depolarizes and the frequency of its action potential increases, providing stretch-induced contraction as occurs with muscle contraction. Stretching of the intestines is crucial for encouraging intestinal motility, and passive exercise of the legs and torso may stretch visceral smooth muscle in the abdomen. After PELT, bowel sounds continued for several minutes. We presume this indicates that the effects of muscular contraction on the bowel lasted for a given period of time.

Significant differences between peristaltic sounds after DBE and at rest were not noted. For up to $20 \mathrm{sec}$ after the technique was halted, the sounds were found to be significantly greater than the sounds at rest. The abdominal viscera are suspended from the diaphragm by the mesentery, and the intestines are stretched by diaphragmatic motion accompanying breathing. In the current study, subjects performed diaphragmatic breathing, presumably resulting in additional stretching of the intestines. Moreover, intestinal motility is closely associated with intra-abdominal pressure, and action that increases intra-abdominal pressure is also crucial for intestinal motility. Diaphragmatic breathing acts to increase intra-abdominal pressure, so stretching and the increase in intra-abdominal pressure due to DBE may have helped to enhance peristaltic sounds. However, the subjects of the current study were healthy adults, and they were able to correctly perform DBE. Patients who have undergone abdominal surgery and elderly patients have difficulty performing DBE, so the types of patients that would be helped by DBE must be studied further.

Peristaltic sounds increased after both of the interventions, so these interventions can be used clinically to treat patients with decreased intestinal motility who are unable to exercise independently or leave their beds due to thera- 
peutic constraints. The present study found that the sounds increased significantly for only $20 \mathrm{sec}$ immediately after both interventions, indicating that both techniques had an immediate effect but no sustained effect. In future clinical settings, techniques should be performed several times for patients with decreased intestinal motility.

\section{ACKNOWLEDGEMENT}

This work was supported by JSPS KAKENHI Grant Number 22792206.

\section{REFERENCES}

1) Amanuma M, Hosoya $T$ : Comparison of the effectiveness of an exercise to encourage peristaltic motion through application of hot compresses to the lumbar region of healthy individuals and individuals with a spinal cord injury. Collected Pap Jpn Nurs Assoc, 2007, 38: 100-102 (in Japanese).

2) Miyajima T, Sato M.: The effectiveness of Miyajima's technique of abdominal compression at encouraging defecation according to bowe sound analysis. Jpn J Nurs Sci, 2005, 25: 2-21 (in Japanese).
3) Emly M, Wilson L, Darby J: Abdominal massage for adults with learning disabilities. Nurs Times, 2001, 97: 61-62. [Medline]

4) Lämås $\mathrm{K}$, Lindholm $\mathrm{L}$, Stenlund $\mathrm{H}$, et al. Effects of abdominal massage in management of constipation: A randomized controlled trial. Int J Nurs Stud, 2009, 46: 759-767. [Medline] [CrossRef]

5) Morizumi M, Hijikata K, Arai R, et al.: Defecation by patients in the terminal stages of gastrointestinal cancer and assistance in sensing abdominal distension: Effectiveness of low-frequency stimulus. Proceedings of the 28th Meeting of the Japanese Nursing Association, 1997: 11-13 (in Japanese).

6) Tanaka N, Tsuchida E: A study of [the effect of] acupressure on defecatory control. Collected Pap Jpn Nurs Assoc, 1999, 30: 104-106 (in Japanese).

7) Politzer JP, Devroede G, Vasseur C, et al.: The genesis of bowel sounds: influence of viscus and gastrointestinal content. Gastroenterology, 1976, 71: 282-285. [Medline]

8) Kagajo Y: Frequency analysis of bowel sounds with an ileus and changes in intestinal tissue oxygen partial pressure. Japanese Journal of Clinical Physiology, 1990: 535-549 (in Japanese).

9) Tomomasa T: Clinical significance of bowel sounds. Gastroenterology, 1999, 28: 257-264 (in Japanese).

10) Iwashiro K: Establishment and use of methods of recording bowel sounds Jpn J Clin Physiol, 1987, 17: 619-631 (in Japanese).

11) Liatsos C, Hadjileontiadis LJ, Mavrogiannis C, et al.: Bowel sounds analysis: A novel noninvasive method for diagnosis of small-volume ascites. Dig Dis Sci, 2003, 48: 1630-1636. [Medline] [CrossRef] 